\title{
Influence of Odd and Even Alkyl Chains on Supramolecular Nanoarchitecture via Self-Assembly of Tetraphenylethylene-Based AIEgens
}

\author{
Mina Salimimarand ${ }^{1}$, Duong Duc La ${ }^{1}$, Sidhanath V. Bhosale ${ }^{2}$, Lathe A. Jones ${ }^{1,3}$ (1) and \\ Sheshanath V. Bhosale ${ }^{1, *}$ \\ 1 School of Science, RMIT University, GPO BOX 2476, Melbourne, VIC 3001, Australia; \\ s3581913@student.rmit.edu.au (M.S.); duc.duong.la@gmail.com (D.D.L.); lathe.jones@rmit.edu.au (L.A.J.) \\ 2 Polymers and Functional Material Division, CSIR-Indian Institute of Chemical Technology, \\ Hyderabad 500 007, India; sidhanath.bhosale@gmail.com \\ 3 Centre for Advanced Materials and Industrial Chemi Stry (CAMIC), RMIT University, GPO Box 2476, \\ Melbourne, VIC 3001, Australia \\ * Correspondence: sheshanath.bhosale@rmit.edu.au or bsheshanath@gmail.com; Tel.: +61-399-252-680
}

Received: 22 September 2017; Accepted: 25 October 2017; Published: 30 October 2017

\begin{abstract}
The Tetraphenylethylene (TPE) based dumbbell shaped molecules TPE-Pi, TPE-Su, TPE-Az, and TPE-Se were synthesised bearing odd-even alkyl chains containing 7, 8, 9 and 10 carbons respectively. These molecules reveal typical Aggregation Induced Emission (AIE) behaviour. The influence of the odd or even alkyl chain length was shown by studying the morphology of self-assembled nanostructures formed in a range of tetrahydrofuran (THF)/water solvent systems. For example, with a water fraction of $80 \%$, TPE derivatives with odd alkyl chains (TPE-Pi and TPE-Az) self-assembled into nanosphere structures, while TPE-Su with 8 alkyl chains formed microbelts and TPE-Se with 10 alkyl chains aggregated into flower-like superstructures. These TPE derivatives also revealed interesting mechanochromic properties upon grinding, fuming and heating, which reveal the importance of molecular stacking in the crystal structure to the luminescent properties of the aggregates .The mechanochromic properties of TPE-Pi, TPE-Su, and TPE-Az were also demonstrated by the process of grounding, fuming, and heating.
\end{abstract}

Keywords: nanostructures; odd-even effect; self-assembly; tetraphenylethylene; mechanochromic properties

\section{Introduction}

Self-assembly of fluorescent $\pi$-conjugated small molecules into well-defined $1 D, 2 D$ and 3D nanostructures has proven to be an interesting strategy for fabricating functional devices with potential applications in chemosensors, biosensing, nanotechnology, biotechnology and optoelectronics [1-8]. A disadvantage of conventional organic fluorophores is that they show aggregation-induced quenching (ACQ) effects due to the formation of excimers or exciplexes [9]. To overcome this drawback, in 2001 Tang et al. investigated, for the first time, the Aggregation-Induced Emission (AIE) phenomenon [10]. AIE fluorophore emits upon aggregation, whereas it is non-emissive when dissolved well in solution [11]. Thus, AIE fluorophores are an ideal building block for fabricating nanostructures with luminescent properties [12]. Tetraphenylethylene (TPE) and its derivatives have shown interesting AIE properties in the solid state due to its unique structure [13]. These AIE fluorophores have been employed to construct self-assembled fibers, nanowires, microrods, microtubes, hollow microspheres, microparticles, microblocks, microparticles, bird nest-like and flower-like structures, and helical morphologies in tetrahydrofuran (THF)/water solvent systems [14-25]. These molecular assemblies 
derive from weak non-covalent interactions, such as hydrogen bonding, electrostatic interactions, $\pi-\pi$ stacking, van der Waals forces and solvophobic interactions. The favourable properties of TPE make it a candidate for applications in chemosensors, biosensors and optoelectronic materials such as field-effect transistors (FETs), organic light emitting diodes (OLEDs) and solar cells [26-33]. The performance of an optoelectronic material is dependent upon the intermolecular interactions and emission efficiencies of the $\pi$-conjugated molecules that it is made from. Therefore, the design, synthesis and development of molecules with high solid-state efficiencies is central to the requirements of materials for optoelectronic applications.

We recently reported the self-assembly of a dumbbell-shaped TPE derivative connecting to an alkyl chain via an amide bond [34]. Scanning Electron Microscopy (FE-SEM) images revealed that this compound had self-organised into a flower-like assembly through a combination of amide-hydrogen bonding and van der Waals interactions. With these results in hand, in order to study the influence that alkyl chain length has on the self-assembly and optical properties of dumbbell shaped TPE molecules, we prepared compounds TPE-Se, TPE-Az, TPE-Su and TPE-Pi, possessing n-decyl, n-nonyl, n-octyl and n-heptyl alkyl chains, respectively (Scheme 1), and studied their morphology after self-assembly and AIE behaviour.

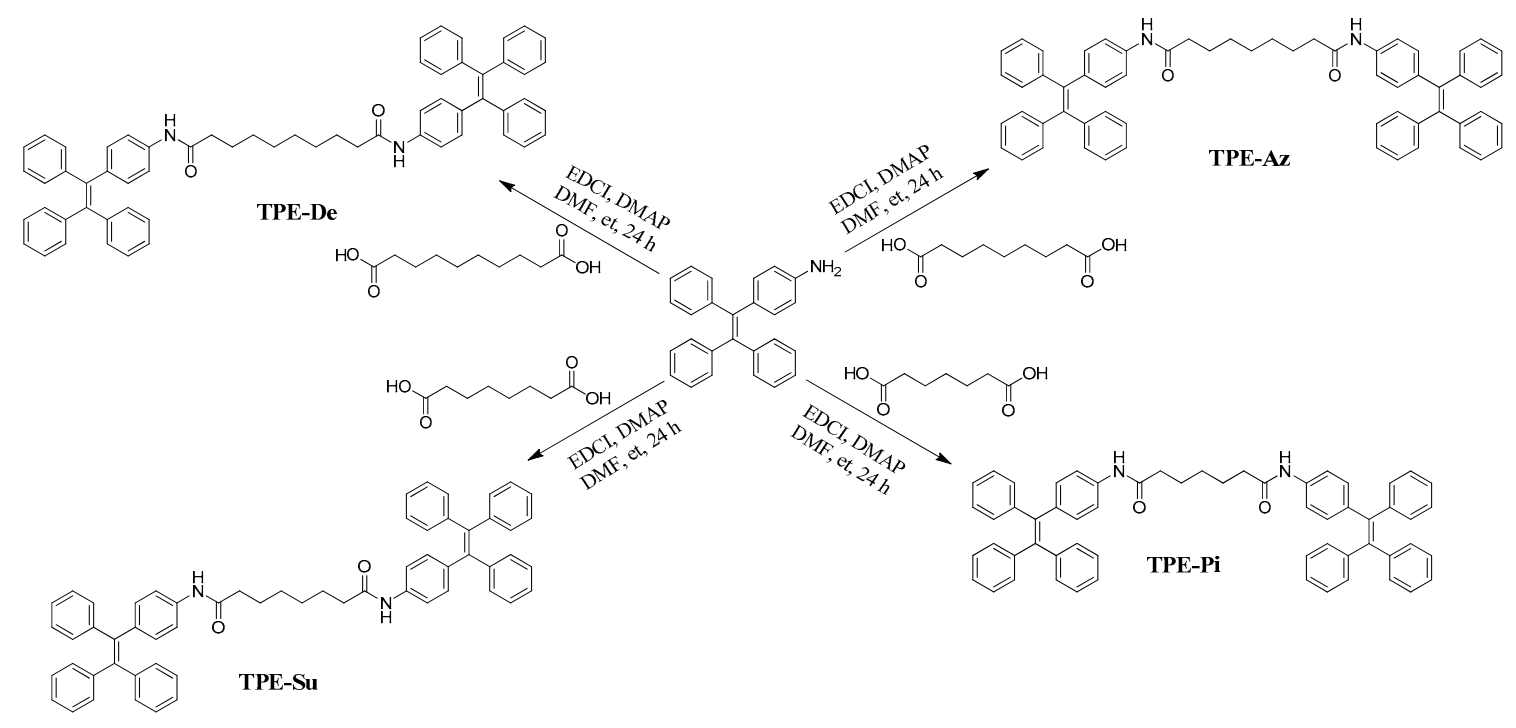

Scheme 1. Synthesis of dumbbell shaped TPE-Se, TPE-Su, TPE-Az and TPE-Pi.

\section{Materials and Methods}

\subsection{Materials}

Decanedioic acid (sebacic acid), octanedioic acid (suberic acid), nonanedioic acid (azelaic acid), heptanedioic acid (pimelic acid), tetraphenylethylene, triethyl amine, EDCI, DMAP and DMF were purchased from Sigma-Aldrich, St. Louis, MO, USA. All reactions were carried out under an argon atmosphere with dry solvents that had been degassed for 10-15 min with argon. Final compounds were purified using silica-gel column chromatography. ${ }^{1} \mathrm{H}-\mathrm{NMR},{ }^{13} \mathrm{C}-\mathrm{NMR}$ spectra were recorded on a Bruker Avance- $300 \mathrm{MHz}$ spectrometer, or a Bruker Advance $75 \mathrm{MHz}$ spectrometer at $300{ }^{\circ} \mathrm{K}$. Chemical shifts (in ppm) were referenced to TMS (Tetramethylsilane) as an internal standard. All measurements were performed in deuterated chloroform $\left(\mathrm{CDCl}_{3}\right)$. IR-Spectra were recorded by using a Thermo Nicolet Nexus 670 spectrometer (New Boston Street, Woburn, MA, USA) in the form of non-hygroscopic $\mathrm{KBr}$ pellets. UV-Vis spectra of pure precursor compounds were recorded using a Shimadzu UV-1800 spectrophotometer (Chiyoda-ku, Tokyo, Japan) at room temperature. Fluorescence emission spectra were collected using a Jobin-Yvon Horiba FluoroMax-4 instrument (Chiyoda-ku, Tokyo, Japan). 


\subsection{Spectroscopic Measurements}

Sample preparation: Stock solutions of TPE-Se, TPE-Su, TPE-Az and TPE-Pi $\left(1 \times 10^{-4} \mathrm{M}\right)$ were prepared in THF. A $0.2 \mathrm{~mL}$ aliquot of the stock solution of each was transferred separately to different volumetric flasks and made up to a $2 \mathrm{~mL}$ volume with respective solvents. The solutions were allowed to equilibrate for $2 \mathrm{~h}$ prior to casting onto a silicon substrate for Scanning Electron Microscopy (SEM) measurements.

UV-Visible Measurements: UV-Vis absorption spectra in mixed solvents were recorded in a Cary-50 in a $1 \mathrm{~cm}$ path length cuvette. The solutions were allowed to equilibrate at room temp for $2 \mathrm{~h}$ before spectral measurements.

Fluorescence Measurements: Fluorescence emission spectra were recorded on a Jobin-Yvon Horiba FluoroMax-4 instrument in a $1 \mathrm{~cm}$ path length cuvette. The solutions were allowed to equilibrate at room temp for $2 \mathrm{~h}$ before spectral measurements.

\subsection{SEM Imaging}

The silicon wafer substrate was cleaned with acetone, ethanol and then Milli $Q$ water. SEM samples were prepared by solvent evaporation onto this silicon wafer and then sputter coated with gold for $10 \mathrm{~s}$ at $0.016 \mathrm{~mA}$ Ar plasma (SPI, West Chester, PA, USA), followed byFE-SEM imaging using a FEI Nova NanoSEM (Hillsboro, OR, USA) operating under high vacuum, which provided direct visualisation of the self-assembled aggregated structures.

\subsection{Mechanochromism Study}

The as-synthesized compounds were ground with a pestle and mortar for $5 \mathrm{~min}$. The fumed samples were prepared by fuming the ground powders with acetone for $60 \mathrm{~s}$. The fumed samples were heated at a temperature of $120^{\circ} \mathrm{C}$ for $10 \mathrm{~min}$.

Synthesis of TPE-Se: This was molecule prepared following our reported paper [34]. Sebacic acid $(0.1 \mathrm{~g}, 0.5 \mathrm{mmol})$ and amino-TPE $(0.407 \mathrm{~g}, 1.08 \mathrm{mmol})$ were added to DMF $(8 \mathrm{~mL})$ at $0{ }^{\circ} \mathrm{C}$ and stirred for $10 \mathrm{~min}$ under nitrogen atmosphere, followed by addition of EDCI $(0.230 \mathrm{~g}, 1.48 \mathrm{mmol})$, DMAP $(0.181 \mathrm{~g}, 1.48 \mathrm{mmol})$ and TEA $(0.2 \mathrm{~mL}, 1.40 \mathrm{mmol})$ at once. The reaction mixture was stirred overnight at room temperature. Reaction completion was checked by TLC analysis. The reaction mixture was then diluted in chloroform followed by washing with $1 \mathrm{M} \mathrm{HCl}$ then $\mathrm{NaHCO}_{3} 10 \%$, and water to remove excess DMF. The crude produced after evaporation was washed with methanol and $2-4$ drops of $\mathrm{HCl}$ and was filtered to remove excess amine, resulting in the white-coloured solid of TPE-1 (0.4 $\mathrm{g}$, $43 \%) .{ }^{1} \mathrm{H}-\mathrm{NMR}\left(300 \mathrm{MHz}, \mathrm{CDCl}_{3}\right) \delta \mathrm{ppm}: 7.13-6.96(\mathrm{~m}, 38 \mathrm{H}), 2.33-2.28(\mathrm{~m}, 4 \mathrm{H}), 1.70-1.58(\mathrm{~m}, 4 \mathrm{H})$, 1.34-1.27 (m, 8H); ${ }^{13} \mathrm{C}-\mathrm{NMR}\left(75 \mathrm{MHz}, \mathrm{CDCl}_{3}\right) \delta$ ppm: 171.1, 143.7, 143.6, 132.0, 131.4, 131.3, 126.4, 126.3, 118.7, 37.7, 29.0, 28.9, 25.4; Mass (ESI) $m / z$ : calculated for $\mathrm{C}_{62} \mathrm{H}_{56} \mathrm{~N}_{2} \mathrm{O}_{2} 860.43$, found: 860.41 $(\mathrm{M})^{+}$; Elemental analysis for $\mathrm{C}_{62} \mathrm{H}_{56} \mathrm{~N}_{2} \mathrm{O}_{2}$ : calculated $=\mathrm{C}, 86.48 ; \mathrm{H}, 6.56 ; \mathrm{N}, 3.25$; found $=\mathrm{C}, 86.53$; $\mathrm{H}, 6.62 ; \mathrm{N}, 3.28$.

Synthesis of TPE-Az: Azelaic acid $(0.1 \mathrm{~g}, 0.5 \mathrm{mmol})$ and amino-TPE $(0.406 \mathrm{~g}, 1.08 \mathrm{mmol})$ were added to DMF $(8 \mathrm{~mL})$ at $0{ }^{\circ} \mathrm{C}$ and stirred for $10 \mathrm{~min}$ under an argon atmosphere, followed by addition of EDCI $(0.230 \mathrm{~g}, 1.48 \mathrm{mmol}), \operatorname{DMAP}(0.181 \mathrm{~g}, 1.48 \mathrm{mmol})$ and TEA $(0.2 \mathrm{~mL}, 1.40 \mathrm{mmol})$ all at once. The reaction mixture was stirred overnight at room temperature. Reaction completion was checked by TLC analysis. The reaction mixture was then diluted in chloroform followed by washing with $1 \mathrm{M} \mathrm{HCl}$, then $\mathrm{NaHCO}_{3}$ $10 \%$, and then water to remove excess DMF. The crude produced after evaporation was washed with methanol and 2-4 drops of $\mathrm{HCl}$ and, after filtration to remove excess amine, the white-coloured solid TPE-Az was obtained (53\%). ${ }^{1} \mathrm{H}-\mathrm{NMR}\left(300 \mathrm{MHz}, \mathrm{CDCl}_{3}\right) \delta \mathrm{ppm:} 7.13-6.96(\mathrm{~m}, 38 \mathrm{H}), 2.33-2.28(\mathrm{~m}, 4 \mathrm{H})$, 1.70-1.58 (m, 4H), 1.34-1.27 (m, 6H); ${ }^{13} \mathrm{C}-\mathrm{NMR}\left(75 \mathrm{MHz}, \mathrm{CDCl}_{3}\right) \delta \mathrm{ppm}: 171.19,142.76 .141 .64,137.38$, $135.32,128.43,127.37,121.75,37.74,29.01,28.90,25.40$. Mass (ESI) $m / z$ : calculated for $\mathrm{C}_{61} \mathrm{H}_{54} \mathrm{~N}_{2} \mathrm{O}_{2}$ 846.41, found $846.40(\mathrm{M})^{+}$; Elemental analysis for $\mathrm{C}_{61} \mathrm{H}_{54} \mathrm{~N}_{2} \mathrm{O}_{2}$ : calculated = C, 86.49; $\mathrm{H}, 6.43 ; \mathrm{N}, 3.31$; found $=\mathrm{C}, 86.53 ; \mathrm{H}, 6.62 ; \mathrm{N}, 3.35$. 
Synthesis of TPE-Su: Suberic acid $(0.1 \mathrm{~g}, 0.5 \mathrm{mmol})$ and amino-TPE $(0.460 \mathrm{~g}, 1.22 \mathrm{mmol})$ were added to DMF ( $8 \mathrm{~mL})$ at $0{ }^{\circ} \mathrm{C}$ and stirred for $10 \mathrm{~min}$ under an argon atmosphere, followed by addition of $\operatorname{EDCI}(0.230 \mathrm{~g}, 1.48 \mathrm{mmol})$, DMAP $(0.181 \mathrm{~g}, 1.48 \mathrm{mmol})$ and TEA $(0.2 \mathrm{~mL}, 1.40 \mathrm{mmol})$ all at once. The reaction mixture was stirred overnight at room temperature. Reaction completion was checked by TLC analysis. The reaction mixture was then diluted in chloroform followed by washing with $1 \mathrm{M}$ $\mathrm{HCl}$ then $\mathrm{NaHCO}_{3} 10 \%$, and finally water to remove excess DMF. The crude product after evaporation was washed with methanol and $2-4$ drops of $\mathrm{HCl}$, and was filtered to remove excess amine, obtaining a white-coloured solid of TPE-Su (52\%). ${ }^{1} \mathrm{H}-\mathrm{NMR}\left(300 \mathrm{MHz}, \mathrm{CDCl}_{3}\right) \delta \mathrm{ppm}: 7.26-6.93(\mathrm{~m}, 38 \mathrm{H})$, 2.35-2.30 (m, 4H), 1.73-1.66 (m, 4H), 1.34-1.27 (m, 4H) ${ }^{13} \mathrm{C}-\mathrm{NMR}\left(75 \mathrm{MHz}, \mathrm{CDCl}_{3}\right) \delta$ ppm: 174.5, 141.0, 140.0, 137.7, 135.1, 128.7, 128.3, 128.1, 127.9, 121.5, 38.3, 27.9, 25.5. Mass (ESI) $m / z$ : calculated for $\mathrm{C}_{60} \mathrm{H}_{52} \mathrm{~N}_{2} \mathrm{O}_{2}$ 832.40, found $832.52(\mathrm{M})^{+}$; Elemental analysis for $\mathrm{C}_{60} \mathrm{H}_{52} \mathrm{~N}_{2} \mathrm{O}_{2}$ : calculated = C, 86.50; $\mathrm{H}, 6.29 ; \mathrm{N}, 3.36$; found $=\mathrm{C}, 86.53 ; \mathrm{H}, 6.32 ; \mathrm{N}, 3.39$.

Synthesis of TPE-Pi: Pimelic acid $(0.1 \mathrm{~g}, 0.5 \mathrm{mmol})$ and amino-TPE $(0.510 \mathrm{~g}, 1.36 \mathrm{mmol})$ were added to DMF ( $8 \mathrm{ml})$ at $0{ }^{\circ} \mathrm{C}$ and stirred for $10 \mathrm{~min}$ under a nitrogen atmosphere, followed by addition of $\operatorname{EDCI}(0.230 \mathrm{~g}, 1.48 \mathrm{mmol})$, DMAP $(0.181 \mathrm{~g}, 1.48 \mathrm{mmol})$ and TEA $(0.2 \mathrm{~mL}, 1.40 \mathrm{mmol})$ all at once. The reaction mixture was stirred overnight at room temperature. Reaction completion was checked by TLC analysis. The reaction mixture was then diluted in chloroform followed by washing with $1 \mathrm{M}$ $\mathrm{HCl}$ then $\mathrm{NaHCO}_{3} 10 \%$, and then water to remove excess DMF. The crude product obtained after evaporation was washed with methanol and 2-4 drops of $\mathrm{HCl}$ and was filtered to remove excess amine, obtaining a white-coloured solid of TPE-Pi (51\%). ${ }^{1} \mathrm{H}-\mathrm{NMR}\left(300 \mathrm{MHz}, \mathrm{CDCl}_{3}\right) \delta$ ppm: 7.11-6.95 (m, 38H), 2.37-2.33 (m, 4H), 1.80-1.73 (m, 4H), 1.34-1.27 (m, 2H); ${ }^{13} \mathrm{C}-\mathrm{NMR}\left(75 \mathrm{MHz}, \mathrm{CDCl}_{3}\right) \delta \mathrm{ppm}:$ $171.57,140.72,140.49,138.18,131.49,131.17,131.12,128.34,128.24,118.77,36.69,28.72,25.34$; Mass (ESI) $m / z$ : calculated for $\mathrm{C}_{59} \mathrm{H}_{50} \mathrm{~N}_{2} \mathrm{O}_{2} 818.38$, found $818.42(\mathrm{M})^{+}$found; Elemental analysis for $\mathrm{C}_{62} \mathrm{H}_{56} \mathrm{~N}_{2} \mathrm{O}_{2}$ : calculated $=\mathrm{C}, 86.52 ; \mathrm{H}, 6.15 ; \mathrm{N}, 3.42$; found $=\mathrm{C}, 86.56 ; \mathrm{H}, 6.18 ; \mathrm{N}, 3.46$.

\section{Results}

The TPE derivatives TPE-Se, TPE-Az, TPE-Su and TPE-Pi were synthesised via amide coupling of amino-TPE and alkyl-dicarboxylic acids catalysed by 1-ethyl-3-(3-dimethylaminopropyl)carbodiimide (EDCI) and 4-dimethylaminopyrimidene (DMAP) in dry DMF (Scheme 2) under a nitrogen atmosphere. The synthesised compounds were fully characterised using ${ }^{1} \mathrm{H}-\mathrm{NMR},{ }^{13} \mathrm{C}-\mathrm{NMR}, \mathrm{ESI}-\mathrm{mass}$ and FTIR spectroscopic techniques (Figures S1 and S4-S13).
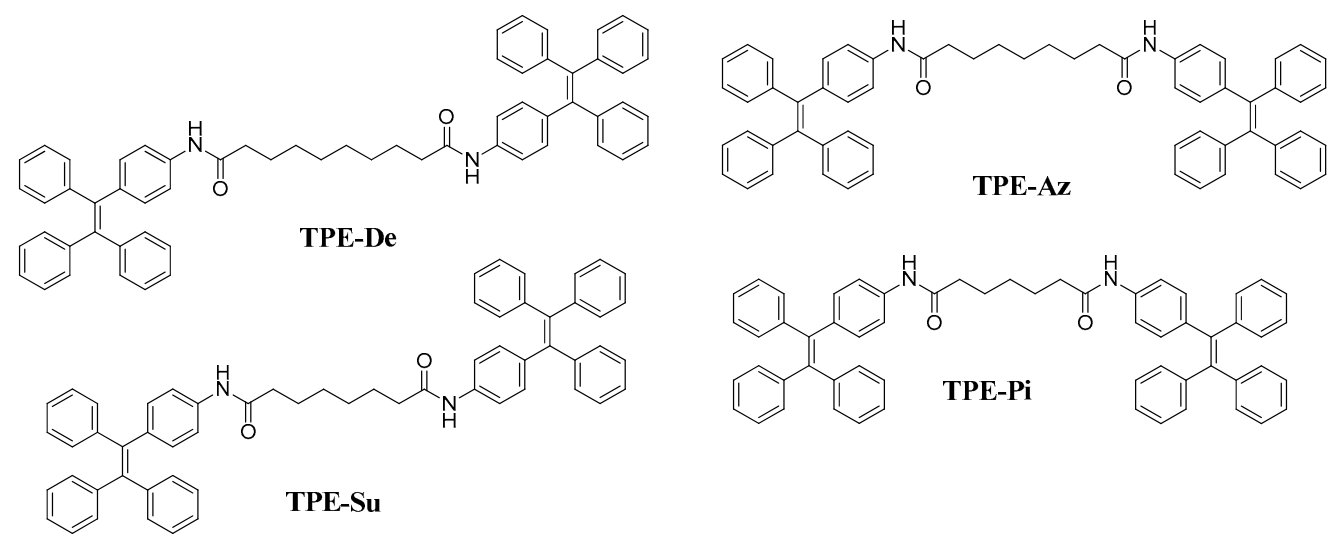

Scheme 2. Structures of dumbbell shaped TPE-Se, TPE-Su, TPE-Az and TPE-Pi derivatives.

The Luminescent properties of TPE-Az, TPE-Su and TPE-Pi under ambient conditions were examined in the THF/water solvent system as shown in Figure 1. In THF, all three TPE derivatives (i.e., TPE-Az, TPE-Su and TPE-Pi) exhibited faint blue emission in solution. Upon incremental addition of water, green fluorescence was first observed at $80 \%$ water. Furthermore, in THF/water 
solution containing $90 \%$ water, we observed strong fluorescence with a higher intensity, indicating an aggregated state of the molecules displaying AIE. The emission colour and intensity change of solution could be readily visualised with the naked eye (Figure 1A-C). The remarkable emission enhancement for TPE-Az, TPE-Su and TPE-Pi is highly reproducible. Here we presume that amide hydrogen bonding and van der Waals interactions are involved in the aggregation process of the TPE derivatives.
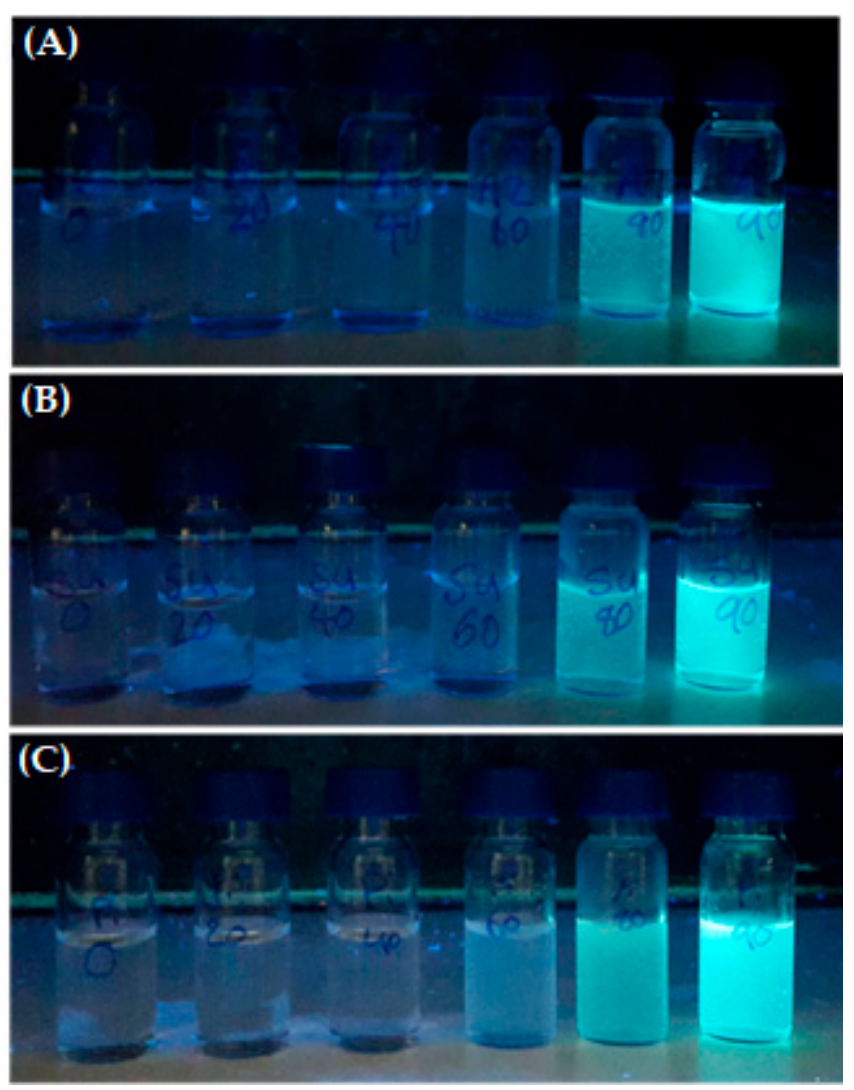

Figure 1. Luminescence properties: photographic images of (A) TPE-Az (B) TPE-Su (C) TPE-Pi in THF-water solvent mixtures with $f_{\mathrm{W}}$ values of $0 \%, 20 \%, 40 \%, 60 \%, 80 \%$ and $90 \%$ respectively, irradiated at $365 \mathrm{~nm}$.

\section{UV-Vis Absorption and Fluorescence Properties}

UV-Vis absorption spectroscopy was employed to investigate the photophysical properties of TPE-Se, TPE-Az, TPE-Su and TPE-Pi (Figure 2). The electronic spectra of $1 \times 10^{-5} \mathrm{M}$ solutions of TPE-Se, TPE-Az and TPE-Pi showed absorption bands at $325 \mathrm{~nm}$ and $260 \mathrm{~nm}$ in THF, corresponding to $\pi-\pi^{*}$ transitions. The UV-Vis spectrum of compound TPE-Su in the same solvent displayed the same absorption maxima at $325 \mathrm{~nm}$ but the shoulder peak at $260 \mathrm{~nm}$ was absent. It should also be noted that the shoulder peak for TPE-Se was batchromically shifted by around $10 \mathrm{~nm}$ to $270 \mathrm{~nm}$ relative to the other compounds. After establishing the absorption properties of $t$ TPE-Se, TPE-Az, TPE-Su and TPE-Pi, the emission properties of the four compounds were measured in THF solution. None of these four compounds showed photoluminescence in THF solution (Figure 3A-C and Figure S2). However, upon incremental addition of water to THF solutions of TPE-Se, TPE-Az, TPE-Su and TPE-Pi, the emission intensity for all four compounds was enhanced. In Figure 3A, the fluorescent emission properties of TPE-Az are shown. Compound TPE-Az was non-fluorescent in THF solution. With the addition of water to the THF solution of TPE-Az, fluorescence emission intensity remained unchanged for $f_{\mathrm{w}}=10-60 \% \mathrm{H}_{2} \mathrm{O} / \mathrm{THF}$ solutions. A slight change in intensity was observed at $f_{\mathrm{w}}=70 \%$. At $f_{\mathrm{w}}=80 \%$, a significant 90 -fold fluorescence emission intensity enhancement was observed. The 
emission peak was split into two peaks at $435 \mathrm{~nm}$ and $535 \mathrm{~nm}$ at $f_{\mathrm{w}}=90 \%$, with an emission enhanced by 120 times compared to pure THF solution. The photoluminescent spectra of TPE-Su are shown in Figure 3B. Once again, a THF solution of TPE-Su did not show any emission. Upon incremental addition of water $(10-60 \%)$ to THF solution, no emission spectral changes were observed. When the water fraction was increased to $70-80 \%$, the emission spectra showed two split peaks at $395 \mathrm{~nm}$ and $535 \mathrm{~nm}$, with a 120-fold increase in emission intensity compared to pure THF. Furthermore, in the THF/water (90\%) mixture the peak at 395 showed a $30 \mathrm{~nm}$ red-shift, and the peak at $535 \mathrm{~nm}$ showed a $40 \mathrm{~nm}$ blue-shift. The new emission bands for TPE-Su at $f_{\mathrm{w}}=90 \%$ appeared at $425 \mathrm{~nm}$ and $495 \mathrm{~nm}$. Similar studies were carried out for compound TPE-Pi in THF with increasing amounts of water (Figure 3C). Emission spectral changes were not observed for an increase in the water fraction between $f_{\mathrm{w}}=10-40 \%$. A further increase in water fraction to $f_{\mathrm{w}}=50 \%$ resulted in a slight increase in emission intensity. With a water fraction of 70\%, a 110-fold emission peak enhancement was observed at $475 \mathrm{~nm}$ compared to emission in pure THF. At $f_{\mathrm{W}}=80 \%$, the emission peak was blue-shifted to $460 \mathrm{~nm}$, and a further increase of water fraction to $90 \%$ led to a split emission peak, at $450 \mathrm{~nm}$ and $525 \mathrm{~nm}$. Figure S2 displays the steady-state fluorescence spectra of the compound TPE-Se in THF and in THF/water solvent mixture. At first, the addition of water (10-50\%) to THF solution resulted in no change in the emission spectra of TPE-Se. But when the water fraction was increased to $60 \%$ to $80 \%$, an increase in emission intensity was observed. At $f_{\mathrm{w}}=90 \%$ the emission peak split into two peaks at $435 \mathrm{~nm}$ and $535 \mathrm{~nm}$ with rapid enhancement of intensity. Both peaks were red-shifted by $25 \mathrm{~nm}$ compared to $f_{\mathrm{w}}=80 \%$. This is a clear demonstration of the AIE behaviour of TPE-Se aggregates via self-assembly. We can conclude that, to achieve maximum emission intensity, a high fraction of water $\left(f_{\mathrm{w}}=80-90 \%\right)$ is required. The addition of a water fraction (0-90\%) to THF solution strongly influences the emission properties of these compounds with respect to peak position and peak intensities (Figure 3D). From fluorescence emission, it can be clearly seen that steady increment of the fluorescent intensity was observed up to $90 \%$ water content, with red-shifted peak intensity. However, with a further increase of the water fraction $>98 \%$, a decrease in intensity was observed, which is due to the larger aggregates and precipitation of self-assembled structures from the solution, as resulting in only a small amount of AIGs being involved in emission. The comparative investigation of emission properties in THF/water clearly suggested that the compound structures TPE-Se, TPE-Az, TPE-Su and TPE-Pi are dramatically affected in $f_{\mathrm{w}}=90 \%$. These results clearly demonstrate that the phenyl ring of TPE around the alkyl chains is restricted in the aggregated state due to the restricted intramolecular rotation (RIR), the mechanism model for the aggregation-induced emission (AIE) phenomenon. The bulky TPE moieties impair the intermolecular $\pi-\pi$ stacking. Thus, the increased FL intensity upon aggregation in higher water content compared to in the dilute THF solutions is because the mechanism for consuming the excitation energy is switched out, similar to earlier report by Tang group [10].

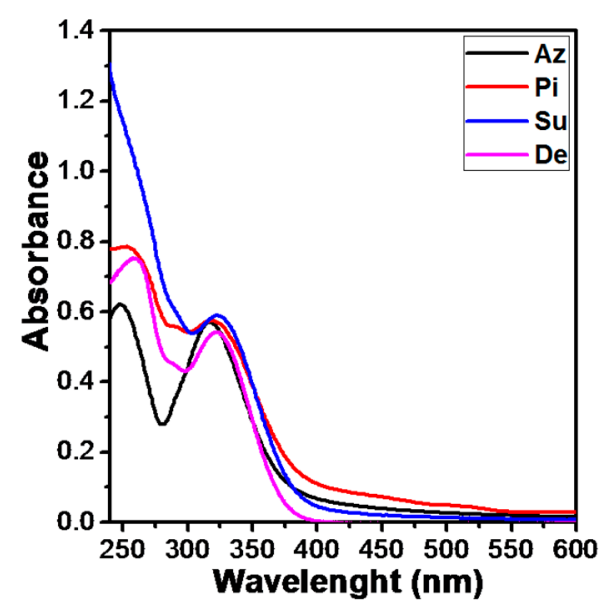

Figure 2. The UV-Vis absorption spectra of TPE-Se, TPE-Az, TPE-Su and TPE-Pi $(10 \mu \mathrm{M})$. 

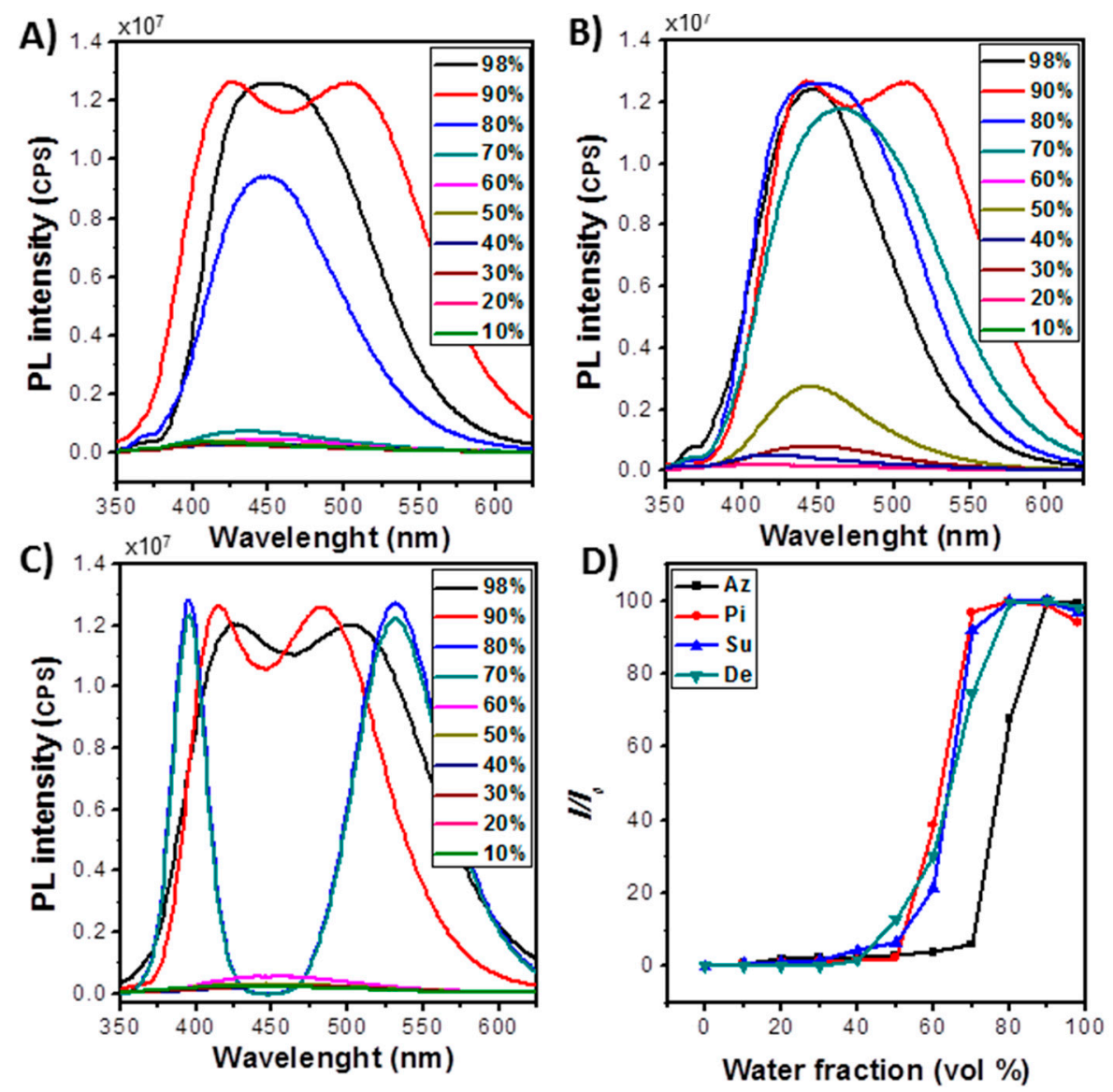

Figure 3. The fluorescence emission spectra of (A) TPE-Az, (B) TPE-Su, (C) TPE-Pi $(10 \mu M)$ in $\mathrm{THF} /$ water at various water fractions $\left(\lambda_{\mathrm{ex}}=365 \mathrm{~nm}\right)$ and (D)The fluorescence intensity changes of TPE-Se, TPE-Az, TPE-Su and TPE-Pi at $475 \mathrm{~nm}$ as a function of water fraction in THF solvent mixture.

In order to elucidate the aggregation behaviour of TPE-Se, TPE-Az, TPE-Su and TPE-Pi in THF/water, as well as the effect of the number of carbons in the alkyl chains on assembly, SEM studies in the presence of various fractions of water/THF were undertaken to observe the morphology of aggregates produced from TPE-Se, TPE-Az, TPE-Su and TPE-Pi, and images are shown in Figure 4 and Figure S2. Figure 4A shows the morphology produced from TPE-Pi aggregation in a THF/water mixture with the water fraction at $80 \%$. It can be clearly seen that, under these conditions, the TPE-Pi (with an odd number of carbons in the alkyl chain of 7) assembled into well-defined nanospheres, with diameters in the range of $200-1000 \mathrm{~nm}$. A further increase in the water fraction $\left(f_{\mathrm{w}}=90 \%\right)$ led to the collapse of the nanospheres into nanobeads, as shown in ESI Figure S3A. When the odd alkyl chains in the TPE compound was increased to 9 (TPE-Az), the morphology of the aggregates in the $\mathrm{THF} / \mathrm{H}_{2} \mathrm{O}$ are remained as spheres, ranging in diameter from 50-500 nm with $f_{\mathrm{w}}=80 \%$ (Figure 4B) and nanobeads with $f_{\mathrm{w}}=90 \%$ (ESI Figure S3B). Interestingly, when the number of carbons in the alkyl chains of the compound are even (TPE-Su with 8 alkyl carbons and TPE-Se with 10 carbons), different morphologies were observed. With the alkyl chain of 8 carbons, microbelts with a width of $5 \mu \mathrm{m}$ and a length of a hundred $\mu \mathrm{m}$ were obtained when the TPE-Su was assembled at $f_{\mathrm{W}}=80 \%$ (Figure 4C). However, similar to TPE-Az and TPE-Pi, only TPE-Su nanobeads were observed upon further addition of water $\left(f_{\mathrm{w}}=90 \%\right.$ ) (ESI Figure S3C). With an alkyl chain of 10 carbons, we recently reported the morphologies of dumbbell shaped TPE-Se in THF/water at different water fractions [34]. Herein, at $f_{\mathrm{w}}=80 \%$, self-assembly of TPE-Se led to flower-like aggregates several micrometers in diameter (Figure 4D). These microflowers were composed of several fractal nanostructures grown from the centre of the flower. These microflowers also disappeared with a water fraction of higher than 
90\% (ESI Figure S3D). TPE-based dumbbell-shaped molecules TPE-Pi, TPE-Su, TPE-Az, and TPE-Se bear odd and even numbers of carbons in the alkyl chains, of 7, 8, 9 and 10, respectively. In particular, odd numbers of carbons ( 7 and 9 ) in the alkyl chains gives mismatching packing, producing particular aggregates. Importantly, even numbers of carbons ( 8 and 10) in the alkyl chains produces a flat, belt-like morphology, and in case of 10 carbons in the alkyl chains produces a flower-like morphology due to the fractal of TPE-Se growing from centre of the flowers. These results are similar to the odd-even effect in supramolecular self-assembly [25], in which odd numbers of carbons in the alkyl chains produces a loosely packed pattern in anti-parallel alignment, and exhibits particular aggregates because of the increased intermolecular interactions. However, even numbers of carbons in the alkyl (perfectly matching) chains pack tightly, and produce fibrils networks with parallel packing.
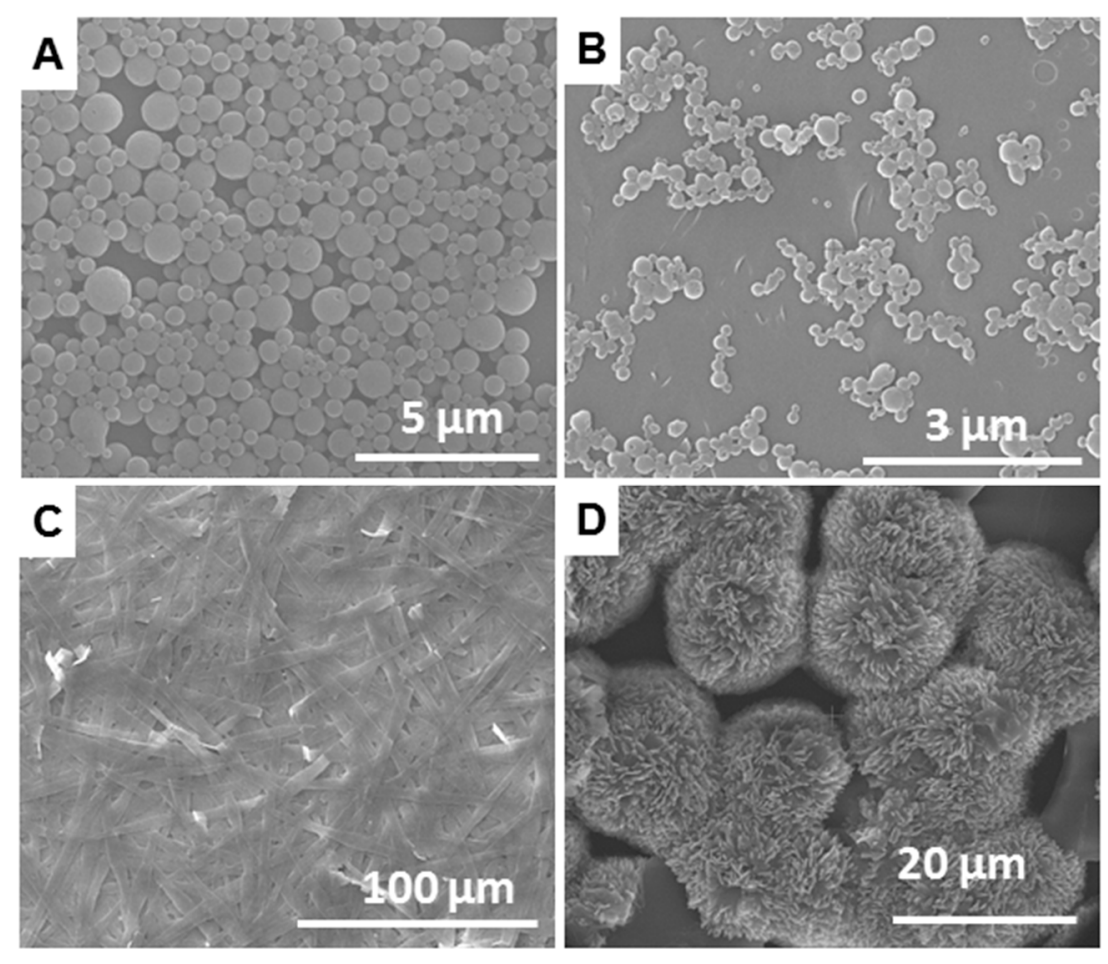

Figure 4. FE-SEM images of aggregates formed from (A) TPE-Pi, (B) TPE-Az, (C) TPE-Su and (D) TPE-Se in THF/water with water fraction of $80 \%$.

The mechanochromic properties of TPE-Pi, TPE-Su, and TPE-Az were also investigated by the process of grinding, fuming, and heating, as shown in Figure 5A-C. The molecular packing of these compounds led to strong emissions in the solid state. This is direct evidence of the AIE effect in these compounds, where the stacking of the TPE moieties as a result of aggregation restricts non-radiative relaxation pathways, and this stacking persists in the solid state. The emission colour and intensity of the powder form are similar to those observed after self-assembly of these compounds in water/THF mixture, suggesting that the structure of the aggregates suspended in solution and the solids were similar. However, the emission intensity of the resulting materials was significantly decreased after grinding. This may be due to the reduction in the crystallite size. Even after fuming with acetone, the ground materials did not revert to their initial luminescent states in terms of either colour or intensity. However, when heating was applied, while the luminescent state was insignificantly altered, the luminescent colours of both TPE-Pi and TPE-Az changed from bright blue to light green. The TPE-Se also showed significant mechanochromic properties, as reported in our previous work [34]. These results confirm that these AIGs are relatively sensitive to molecular stacking in the crystal structure. 
A)
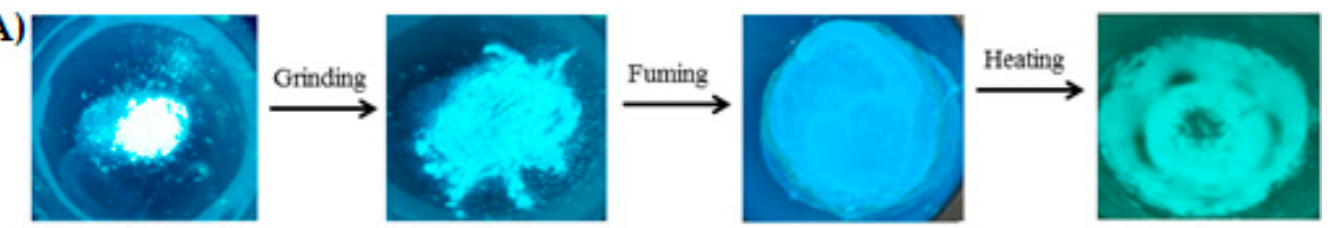

B)
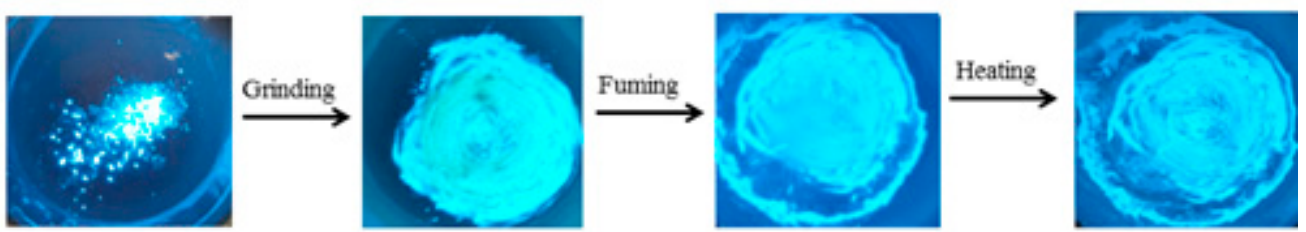

C)
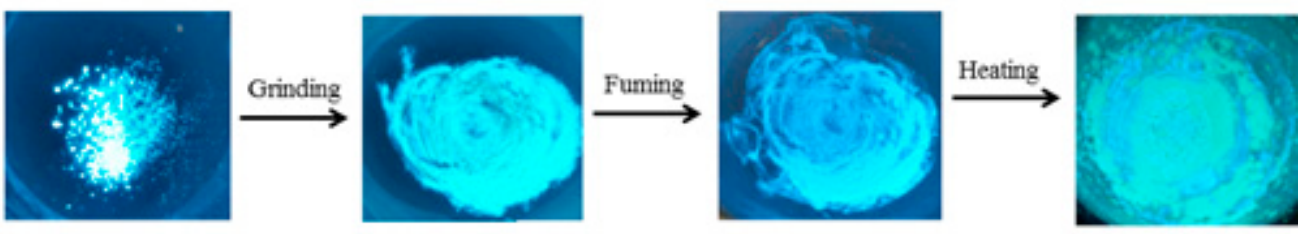

Figure 5. The mechanochromic properties of (A) TPE-Pi, (B) TPE-Su, and (D) TPE-Az, showing the luminescence changing of these compounds after grinding, fuming and heating.

\section{Conclusions}

Tetraphenylethylene (TPE)-based dumbbell-shaped molecules TPE-Pi, TPE-Su, TPE-Az, and TPE-Se bearing an odd-even number of carbons in alkyl chains of 7, 8, 9 and 10, respectively, were synthesised. The solvophobic-controlled self-assembly, as well as luminescent properties of these compounds, were studied in detail. Similar to other TPE derivatives, TPE-Pi, TPE-Su, TPE-Az, and TPE-Se showed typical AIE behaviour, being non-emissive in THF solution and emitting strongly in THF/water mixture due to self-assembly of aggregates. With a water fraction of $80 \%$, while TPE derivatives with odd alkyl chains (TPE-Pi and TPE-Az) self-assembled into nanosphere structures, TPE-Su with 8 carbons in the alkyl chain formed microbelts and TPE-Se with 10 carbons in the alkyl chain aggregated into flower-like superstructures. These TPE derivatives also revealed interesting mechanochromic properties upon grinding, fuming and heating, revealing the sensitivity of luminescent properties to molecular stacking in the crystal structure and crystallite size. The results described in this paper can be employed to pursue further strategies in component design and self-assembly theory that go beyond simple morphologies.

Supplementary Materials: The following are available online at http://www.mdpi.com/2076-3417/7/11/1119/s1, The following ESI are available online for compounds (A) TPE-Pi, (B) TPE-Az, (C) TPE-Su and (D) TPE-Se. Figure S1: FTIR, Figure S2: Fluorescence emission spectra of TPE-Se; Figure S3: FE-SEM images of the suspension of (A) TPE-Pi, (B) TPE-Az, (C) TPE-Su and (D) TPE-Se in THF/water with a water fraction of $90 \%$.

Acknowledgments: S.V.B. (RMIT) acknowledges the Australian Research Council for financial support under a Future Fellowship Scheme (FT110100152). S.V.B. (IICT) is grateful for financial support from the SERB (DST) SB/S1/IC-009/2014, New Delhi, India. The authors acknowledge the facilities, and the scientific and technical assistance of the Australian Microscopy \& Microanalysis Research Facilities (MMRF) at RMIT University.

Author Contributions: M.S. performed synthesis and structure determination using spectroscopic techniques along with UV-Vis and fluorescence spectroscopy. D.D.L. performed surface-based assembly to visualise the nanostructures by SEM. S.V.B (IICT) drafted the first draft of the manuscript, and L.A.J. corrected the manuscript and co-supervised the D.D.L. S.V.B. (RMIT) directed the research, interpreted and analysed the data, and wrote the full manuscript. All co-authors reviewed the manuscript.

Conflicts of Interest: The authors declare no conflict of interest.

\section{References}

1. Lehn, J.M. Supramolecular Chemistry: Concepts and Perspectives; VCH: Weinheim, Germany, 1995.

2. Zang, L.; Che, Y.; Moore, J.S. One-dimensional self-assembly of planar $\pi$-conjugated molecules: Adaptable building blocks for organic nanodevices. Acc. Chem. Res. 2008, 41, 1596-1608. [CrossRef] [PubMed] 
3. Kaeser, A.; Schenning, A.P.H.J. Fluorescent nanoparticles based on self-assembled $\pi$-conjugated systems. Adv. Mater. 2010, 22, 2985-2997. [CrossRef] [PubMed]

4. Zhao, Y.S.; Fu, H.; Peng, A.; Ma, Y.; Liao, Q.; Yao, J. Construction and optoelectronic properties of organic one-dimensional nanostructures. Acc. Chem. Res. 2010, 43, 409-418. [CrossRef] [PubMed]

5. Chakrabarty, R.; Mukherjee, P.S.; Stang, P.J. Supramolecular coordination: Self-assembly of finite two-and three-dimensional ensembles. Chem. Rev. 2011, 111, 6810-6918. [CrossRef] [PubMed]

6. Magginia, L.; Bonifazi, D. Hierarchised luminescent organic architectures: Design, synthesis, self-assembly, self-organisation and functions. Chem. Soc. Rev. 2012, 41, 211-241. [CrossRef] [PubMed]

7. Ren, C.; Zhang, J.; Chen, M.; Yang, Z. Self-assembling small molecules for the detection of important analytes. Chem. Soc. Rev. 2014, 43, 7257-7266. [CrossRef] [PubMed]

8. Babu, S.S.; Praveen, V.K.; Ajayaghosh, A. Functional $\pi$-gelators and their applications. Chem. Rev. 2014, 114, 1973-2129. [CrossRef] [PubMed]

9. Birks, J.B. Photophysics of Aromatic Molecules; Wiley: New York, NY, USA, 1970.

10. Luo, J.; Xie, Z.; Lam, J.W.Y.; Cheng, L.; Chen, H.; Qiu, C.; Kwok, H.S.; Zhan, X.; Liu, Y.; Zhu, D.; et al. Aggregation-induced emission of 1-methyl-1,2,3,4,5-pentaphenylsilole. Chem. Commun. 2001, 18, 1740-1741. [CrossRef]

11. Mei, J.; Hong, Y.; Lam, J.W.Y.; Qin, A.; Tang, Y.; Tang, B.Z. Aggregation-induced emission: The whole is more brilliant than the parts. Adv. Mater. 2014, 26, 5429-5479. [CrossRef] [PubMed]

12. Hong, Y.; Lam, J.W.Y.; Tang, B.Z. Aggregation-induced emission: Phenomenon, mechanism and applications. Chem. Commun. 2009, 29, 4332-4353. [CrossRef] [PubMed]

13. Hong, Y.; Lam, J.W.Y.; Tang, B.Z. Aggregation-induced emission. Chem. Soc. Rev. 2011, 40, 5361-5388. [CrossRef] [PubMed]

14. Zhao, Q.; Zhang, S.; Liu, Y.; Mei, J.; Chen, S.; Lu, P.; Qin, A.; Ma, Y.; Sun, J.Z.; Tang, B.Z. Tetraphenylethenyl-modified perylenebisimide: Aggregation-induced red emission, electrochemical properties and ordered microstructures. J. Mater. Chem. 2012, 22, 7387-7394. [CrossRef]

15. Shen, X.Y.; Wang, Y.J.; Zhao, E.; Yuan, W.Z.; Liu, Y.; Lu, P.; Qin, A.; Ma, Y.; Sun, J.Z.; Tang, B.Z. Effects of substitution with donor-acceptor groups on the properties of tetraphenylethene trimer: Aggregation-induced emission, solvatochromism, and mechanochromism. J. Phys. Chem. C 2013, 117, 7334-7347. [CrossRef]

16. Rananaware, A.; Bhosale, R.S.; Ohkubo, K.; Patil, H.; Jones, L.A.; Jackson, S.L.; Fukuzumi, S.; Bhosale, S.V.; Bhosale, S.V. Tetraphenylethene-Based Star Shaped Porphyrins: Synthesis, Self-assembly, and Optical and Photophysical Study. J. Org. Chem. 2015, 80, 3832-3840. [CrossRef] [PubMed]

17. Feng, H.-T.; Song, S.; Chen, Y.-C.; Shen, C.-H.; Zheng, Y.-S. Self-assembled tetraphenylethylene macrocycle nanofibrous materials for the visual detection of copper(II) in water. J. Mater. Chem. C 2014, 2, 2353-2359. [CrossRef]

18. Niu, C.; Zhao, L.; Fang, T.; Deng, X.; Ma, H.; Zhang, J.; Na, N.; Han, J.; Ouyang, J. Color- and morphology-controlled self-assembly of new electron-donor-substituted aggregation-induced emission compounds. Langmuir 2014, 30, 2351-2359. [CrossRef] [PubMed]

19. Han, M.; Cho, S.J.; Norikane, Y.; Shimizu, M.; Kimura, A.; Tamagawa, T.; Seki, T. Multistimuli-responsive azobenzene nanofibers with aggregation-induced emission enhancement characteristics. Chem. Commun. 2014, 50, 15815-15818. [CrossRef] [PubMed]

20. Song, S.; Zheng, H.-F.; Feng, H.-T.; Zheng, Y.-S. Microtubes and hollow microspheres formed by winding of nanoribbons from self-assembly of tetraphenylethylene amide macrocycles. Chem. Commun. 2014, 50, 15212-15215. [CrossRef] [PubMed]

21. Liu, J.; Su, H.; Meng, L.; Zhao, Y.; Deng, C.; Ng, J.C.Y.; Lu, P.; Faisal, M.; Lam, J.W.Y.; Huang, X.; et al. What makes efficient circularly polarised luminescence in the condensed phase: Aggregation-induced circular dichroism and light emission. Chem. Sci. 2012, 3, 2737-2747. [CrossRef]

22. La, D.D.; Bhosale, S.V.; Jones, L.A.; Bhosale, S.V. Tetraphenylethylene-based AIE-Active Probes for Sensing Applications. ACS Appl. Mater. Interfaces 2017. [CrossRef] [PubMed]

23. Rananaware, A.; La, D.D.; Jackson, S.M.; Bhosale, S.V. Construction of a highly efficient near-IR solid emitter based on naphthalene diimide with AIE-active tetraphenylethene periphery. RSC Adv. 2016, 6, 16250-16255. [CrossRef] 
24. Li, H.; Zheng, X.; Su, H.; Lam, J.W.Y.; Wong, K.S.; Xue, S.; Huang, X.; Huang, X.; Li, B.S.; Tang, B.Z. Synthesis, optical properties, and helical self-assembly of a bivaline-containing tetraphenylethene. Sci. Rep. 2016, 6, 19277. [CrossRef] [PubMed]

25. Anuradha, A.; La, D.D.; Al Kobaisi, M.; Bhosale, S.V. Right handed chiral superstructures from achiral molecules: Self-assembly with a twist. Sci. Rep. 2015, 5, 15652.

26. Rananaware, A.; Bhosale, R.S.; Patil, H.; Al Kobaisi, M.; Abraham, A.; Shukla, R.; Bhosale, S.V.; Bhosale, S.V. Precise aggregation-induced emission enhancement via $\mathrm{H}^{+}$sensing and its use in ratiometric detection of intracellular $\mathrm{pH}$ values. RSC Adv. 2014, 4, 59078-59082. [CrossRef]

27. Dong, Y.; Lam, J.W.Y.; Qin, A.; Liu, J.; Li, Z.; Tang, B.Z. Aggregation-induced emissions of tetraphenylethene derivatives and their utilities as chemical vapour sensors and in organic light-emitting diodes. Appl. Phys. Lett. 2007, 91, 011111. [CrossRef]

28. La, D.D.; Anuradha, A.; Kaur Hundal, A.; Jones, L.A.; Bhosale, S.V. pH-Dependent self-assembly of water-soluble sulphonate-tetraphenylethylene with aggregation-induced emission. Supramol. Chem. 2017. [CrossRef]

29. Mei, J.; Leung, N.L.; Kwok, R.T.; Lam, J.W.; Tang, B.Z. Aggregation-induced emission: Together we shine, united we soar. Chem. Rev. 2015, 21, 11718-11940. [CrossRef] [PubMed]

30. Zhang, H.; Li, H.; Wang, J.; Sun, J.; Qin, A.; Tang, B.Z. Axial chiral aggregation-induced emission luminogens with aggregation-annihilated circular dichroism effect. J. Mater. Chem. C 2015, 3, 5162-5166. [CrossRef]

31. Liu, X.; Jiao, J.; Jiang, X.; Li, J.; Cheng, Y.; Zhu, C. A tetraphenylethene-based chiral polymer: An AIE luminogen with high and tunable CPL dissymmetry factor. J. Mater. Chem. C 2013, 1, 4713-4719. [CrossRef]

32. Zhao, Z.; Lam, J.W.; Tang, B.Z. Tetraphenylethene: A versatile AIE building block for the construction of efficient luminescent materials for organic light-emitting diodes. J. Mater. Chem. 2012, 22, 23726-23740. [CrossRef]

33. Rananaware, A.; Gupta, A.; Lo, J.; Bilic, A.; Jones, L.; Bhargava, S.; Bhosale, S.V. A four-directional non-fullerene acceptor based on tetraphenylethylene and diketopyrrolopyrrole functionalities for efficient photovoltaic devices with a high open-circuit voltage of 1.18 V. Chem. Commun. 2016, 52, 8522-8525. [CrossRef] [PubMed]

34. Salimimarand, M.; La, D.D.; Al Kobaisi, M.; Bhosale, S.V. Flower-like superstructures of AIE-active tetraphenylethylene through solvophobic controlled self-assembly. Sci. Rep. 2017, 7, 42898. [CrossRef] [PubMed]

(C) 2017 by the authors. Licensee MDPI, Basel, Switzerland. This article is an open access article distributed under the terms and conditions of the Creative Commons Attribution (CC BY) license (http:/ / creativecommons.org/licenses/by/4.0/). 